\title{
Flow at interfaces: A new device for $x$-ray surface scattering investigations
}

\author{
J.-F. Moulin a) \\ Physik-Department, TU München, LS E13, James-Franck-Str. 1, 85747 Garching, Germany \\ S. V. Roth \\ HASYLAB at DESY, Notkestr. 85, 22603 Hamburg, Germany \\ P. Müller-Buschbaum \\ Physik-Department, TU München, LS E13, James-Franck-Str. 1, 85747 Garching, Germany
}

(Received 10 August 2007; accepted 29 October 2007; published online 16 January 2008)

\begin{abstract}
A fluidic cell based setup is described which allows for microbeam grazing incidence small angle $\mathrm{x}$-ray scattering characterization of the interface between a solid substrate and a flowing liquid. This cell can potentially be used to study in situ a wide variety of systems ranging from synthetic and natural colloids to biological molecules. The selected channel geometry enables the characterization of the solid-liquid interface during mixing of different solutions. As a proof of concept, measurements on an aqueous gold nanoparticle solution in contact with a glass surface are presented that show that the structure at the interface can be probed during flow. (0) 2008 American Institute of Physics. [DOI: 10.1063/1.2816220]
\end{abstract}

\section{INTRODUCTION}

Flow of a liquid upon a solid surface is controlled by interactions on different length scales: the interactions between the constituents of the liquid and the solid substrate on the atomic scale as well as the substrate morphology in terms of corrugation, roughness, and topography at large length scales give rise to a rich, system-specific behavior.

Microfluidic devices which manipulate microquantities of liquid solutions allow for substantial improvement of the efficiency of chemical processes, and the exact control of mixing and separation processes during fluid flow in confined geometries is thereby essential for many applications as microanalytics or high throughput devices and reactors ${ }^{1-5}$ as well as for analytical applications, such as chromatography. For the design of new microfluidic devices and lab-on-chip systems the understanding of nano- and microfluidics of multicomponent fluids is crucial since in chemical reactions at least two reaction partners are involved. Therefore, it is important to study the transport, mixing, demixing, separation, filtration, or sorting operations of starting substances and reaction products during liquid flow. For transport and mixing a variety of tools as pumps ${ }^{6,7}$ valves,${ }^{6}$ channels, ${ }^{8,9}$ sensors, ${ }^{10}$ and mixers ${ }^{11-13}$ on the micro- or even nanoscale were developed for a variety of substances. Also more complicated operations such as separation, filtration, and sorting objects were performed ${ }^{14}$ using tailored complex devices for very special tasks as nanopore filters coated with polymer brushes, ${ }^{15}$ reaction vessels with colloidal particles, ${ }^{16}$ and magnetic particle matrices for DNA separation. ${ }^{17}$

In order to understand fundamental problems such as pattern formation, phase separation, or mixing in confined geometries $^{18,19}$ and to use this knowledge to specifically de-

a) Now at GKSS Forschungszentrum Geesthacht $\mathrm{GmbH}$, Institut für Werkstoffforschung, Lichtenberg-Str, 4 D85747 Garching (Germany). sign systems, structural insight about the ever more important interfaces is needed. This characterization should yield information about very different length scales, from molecular dimensions to mesoscopic and up to microscopic distances (the size of the actual container).

Up to now, mostly microscopy based experimental techniques have been employed to investigate the behavior of fluids in channels and confined near surfaces. Although crucial, these techniques should be complemented with others to cover a larger range of characteristic lengths. Moreover, complementary methods should be sensitive to composition fluctuations and provide a direct statistically relevant information via a representative sampling. All these features are clearly within reach of scattering techniques. In fact, small angle scattering (either of neutrons or x-rays) provides information such as object shapes, sizes, and characteristic correlation lengths between objects. ${ }^{20}$ Applying scattering to fluids is not at all a new idea but most of the existing work has focused either on bulk systems using measurements in transmission geometry ${ }^{21,22}$ or specular reflectivity. ${ }^{23,24}$ In transmission geometry no information about the fluid-solid interface is provided and specular reflectivity yields the density profile in the direction normal to the interface. This projection is of course very useful to elucidate the layering of the structure parallel to the substrate ${ }^{24}$ but leaves us with no information concerning the actual organization within the inplane direction. Nevertheless, it is clearly of utmost importance to get information about spatial correlations within planes parallel to the interface (e.g., to describe an ordered surface such as the one formed via phase separation of incompatible polymer blends). Grazing incidence x-ray scattering (GIXS) provides such information on parallel structures. ${ }^{25-28}$ Due to the peculiar geometry of the experiment, it offers a dramatic enhancement of the signal coming 
from the probed interface, without significantly sensing the overlaying bulk of solution (which is accessible via more conventional x-ray scattering). ${ }^{29}$

GIXS has gained a lot of interest in the past years since it enables to study interfacial structures in a noninvasive and nondestructive way. ${ }^{30}$ In particular, when the scattered beam is collected in a small angle (GISAXS) or ultrasmall angle configuration it enables the characterization of surface order on large or very large scales: for example, structures up to $20 \mu \mathrm{m}$ can be detected at state of the art beamlines. ${ }^{31}$ Using focusing optics, available at these beamlines, ${ }^{32}$ the high reciprocal space resolution can be coupled to a certain extent with a real-space resolution in the $1-50 \mu \mathrm{m}$ range, thus allowing to perform a scanning analysis of the samples.

Nevertheless, up to now, these techniques have not been applied to investigate systems under flow. In order to perform a GISAXS experiment on a fluidic system, a setup is required which optimizes the useful signal by minimizing the absorption and parasitic scattering from the cell itself while still ensuring proper sealing in combination with a well defined geometry. Recently, Reich et al. proposed a special fluidic cell design for specular x-ray reflectivity with $20 \mathrm{keV}$ photon energy. ${ }^{33}$ This fluidic cell is based on a modification of a relatively large commercial plastic cell which exhibits a $5 \mathrm{~mm}$ wide channel. Within this article we describe our efforts in developing a fluidic cell which allows for GISAXS measurements to target the characterization of interfacial flow in channels of reduced size. In order to achieve this goal, the device and, in particular, its walls have to be downsized with respect to conventional fluidic cells. In the grazing incidence geometry of a GISAXS experiment the beam size is strongly elongated along the beam direction due to the projection of the beam diameter onto the sample surface. As a compromise between this footprint effect causing the necessary beam size reduction and the achievable channel width downsizing we decided to develop a $1 \mathrm{~mm}$ wide channel system. Such system is compatible with a relaxed microfocusing of the $\mathrm{x}$ rays (lateral beam size in the range of $30 \mu \mathrm{m})$. In addition, the developed design provides the opportunity to mix various solutions (or, in reverse flow mode, to separate two streams of solution) and thus to perform kinetic experiments. Different mixing or demixing times are accessible simply by scanning various positions of the cell with respect to the inlet and outlet.

After the description of the fluidic cell design, the cell testing, both in terms of fluidics and as a scattering chamber are presented. As a model system, results obtained from the characterization of a gold nanoparticle solution flow over a glass substrate are shown. The discussion of the results and the perspectives opened by this setup are followed by a summary.

\section{CELL DESIGN}

The constraints were to develop a general and flexible design that could easily be adjusted to specific needs in terms of different substrates exposed to flow and would provide multiple inlets in order to characterize mixing or demixing of

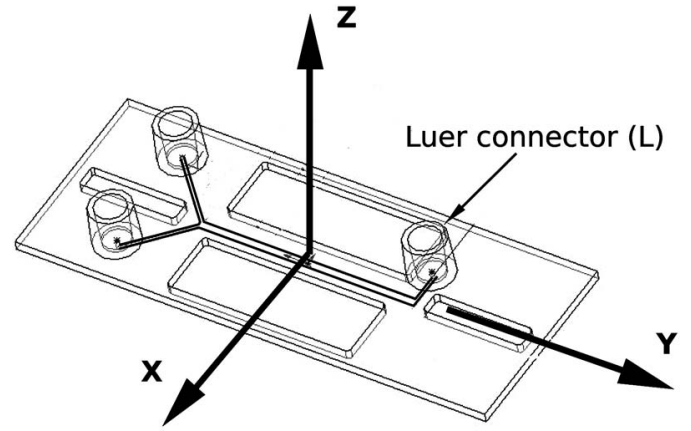

FIG. 1. Design layout of the TOPAS part of the fluidic cell. The asymmetric design with two inlets and one outlet (or vice versa) enables mixing of fluids. The channel system itself is shown in dark gray. Included is a realspace coordinate system.

different fluids. The main idea was to create a complete channel system with its input and output connectors.

The top window of the cell was kept thin and transparent to visible light in order to allow for optical characterization. The whole channel is brought in contact with the surface of a variable flat substrate (e.g., a glass surface or silicon wafer). The complete assembly (top surface with channel system and substrate) is then attached on the x-ray goniometer via a rigid metal clamp. In order to prevent contamination of the investigated substrate surface via diffusion of adhesive, no glue is used to seal the channel.

We chose to use the same material as that of Reich et $a l^{33}$ for the cell body [top surface with channel system (TOPAS)], which is a commercially available cyclic olefin based copolymer resistant to polar organic solvents and water. This material can be easily machined and thermally bonded to itself. It has a relatively low absorption in the $\mathrm{x}$-ray range (the attenuation length of TOPAS is $\xi_{\text {TOPAS }}$ $=4.9 \mathrm{~mm}$ at $10 \mathrm{keV}$, the photon energy we used for our synchrotron experiment). Moreover it is transparent to visible light which enables optical characterization of the flow via microscopy.

As mentioned before, in order to keep absorption low, the wall thickness has to be kept to the strict minimum that ensures proper sealing. A wall thickness of $0.5 \mathrm{~mm}$ was determined as an optimum value. The channel width was chosen to be $1 \mathrm{~mm}$, which provides a reasonable trade-off in terms of transmission and use of the beam's footprint. The transmission of the beam under grazing incidence conditions can then be well approximated by $e^{-2}\left(t_{\text {wall }} / \xi_{\text {TOPAS }}\right)=0.81$ for the empty cell and $e^{-2}\left(t_{\text {wall }} / \xi_{\text {TOPAS }}\right) e^{-}\left(t / \xi_{\text {water }}\right)=0.25$ for a water filled channel $\left(t_{\text {wall }}\right.$ is the thickness of the cell walls, and $\xi_{\text {TOPAS }}$ and $\xi_{\text {water }}$ are the absorption length of TOPAS and water, respectively). This relatively short path length ensures that our design will still be usable to investigate more absorbing solutions. The footprint length for our setup is $7.45 \mathrm{~mm}$ under the experimental conditions used, and the small channel size makes it easy to align the cell in the beam.

This channel was machined in a TOPAS foil (length of $75 \mathrm{~mm}$, width of $25 \mathrm{~mm}$, and thickness of $1 \mathrm{~mm}$ ) which was then bonded to a $200 \mu \mathrm{m}$ thick TOPAS window. The channel has a Y shape (see Fig. 1) in such a way that mixing (or demixing) of solutions can be realized. The thin channel 


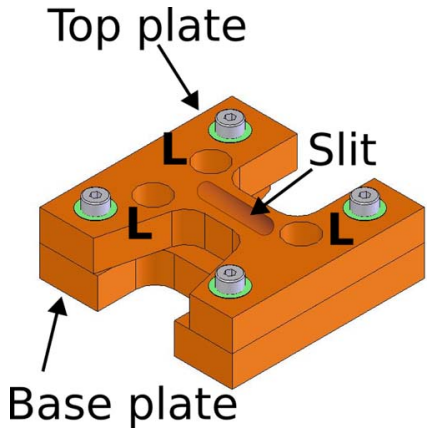

FIG. 2. (Color online) Design layout of the metal clamp which allows for a sealing of the cell body to various substrates without using glue. The openings for the Luer connectors are shown (L) as well as the slit for optical observation.

walls are quite fragile and can easily be twisted during sample preparation. We thus reinforced the structure by leaving two bridges that can be easily cut away once the setup is locked in the clamp (shown in Fig. 2). The input and output connectors are standard Luer plugs also made of TOPAS and bonded to the top window after drilling of the inlet and outlet holes.

The clamp used to assemble the cell body and the substrate is made of $8 \mathrm{~mm}$ thick stainless steel for reasons of rigidity. Holes were drilled to accommodate the Luer connectors. A slit in the top plate was added to enable the optical observation of the flow. On the one hand, the notches on the side of the clamps are intended to limit the shadowing of the $\mathrm{x}$-ray beam and to minimize the risk of parasitic scattering, and on the other hand, to enable optical observation of the cell walls in order to check for leaks.

Mounting and sealing the cell are the critical steps of the fluidic experiments. Within the work presented here, we used $1 \mathrm{~mm}$ thick microscope glass slides as a substrate. The glass surfaces were cleaned to remove any contamination which could affect surface properties and sealing quality, ${ }^{34}$ The TOPAS channel was then carefully placed on the substrate, taking care to avoid torsion or scratching of the surfaces. In the next step the clamp was closed and slowly tightened while maintaining uniform pressure. The torque applied to each screw, as measured with a dynamometric key, was typically of $6 \mathrm{~N} \mathrm{~m}$.

Injection of the filtered solutions was performed using a syringe pump (model PHD 2000 infuse/withdraw from Harvard Apparatus). The syringes were connected to the cell via $1.8 \mathrm{~mm}$ inner diameter silicone tubes and Luer adapters.

\section{CELL TESTING AND PERFORMANCE}

\section{A. Controlling the mixing profile as a function of speed}

In order to check for leaks and control the stability of the flow achieved in the channel, we performed a simple mixing experiment with pure water and a dye solution. The channel was observed by means of a charge coupled device (CCD) camera placed above the slit of the top clamp. Images were recorded at different flow rates, and the mixing profile of the dye into water was determined from these images. The extraction of the profile was performed by comparing the in- tensity of the blue channel of the CCD image to the sum of the three channels (red, green, and blue). This gives a measure of the concentration of our blue dye at each sampled position in the channel. At the highest flow speed $(8300 \mu \mathrm{m} / \mathrm{s})$ interdiffusion of the dye and water is too slow to allow mixing within the channel and a clear front is observed [Fig. 3(a), top]. This translates into indistinguishable color profiles at all cutting positions along the channel [Fig. 3(b), top]. The profiles are sharp on the optical scale and centered at the middle of the channel. For slower flow rates, diffusion takes place and the color profiles measured along the channel vary accordingly from a sharp step, evidence of no mixing, to a much broader one, indicating a more uniform dye distribution. For the slowest flow rate $(8.3 \mu \mathrm{m} / \mathrm{s})$, the dye concentration is observed to be almost constant over the whole channel, indicating complete and uniform mixing [Figs. 3(a) and 3(b), bottom]. Observation of a constantly laminar flow regime gave us the assurance that the influence of the machined surface corrugation upon flow is negligibly small at the used flow rates.

\section{B. Microbeam GISAXS measurements}

Microbeam grazing incidence small angle x-ray scattering $(\mu \mathrm{GISAXS})$ testing of the cell was performed at beamline BW4 at HASYLAB (DESY) in Hamburg. The wavelength was $0.138 \mathrm{~nm}$ and moderate microbeam focusing was achieved using beryllium compound refractive lenses (beam size of $65 \times 35 \mu \mathrm{m}^{2}$ ). The two dimensional (2D) detector, a MAR CCD camera with $79 \mu \mathrm{m}$ pixel size, was placed at $1.9 \mathrm{~m}$ from the sample. The complete beam path was under high vacuum except for an air gap sealed by two kapton windows where the flow cell was located. The incident angle was set above the critical angle for total external reflection from the glass substrate $\alpha_{i}=0.5^{\circ}$. The total counting time for each image shown here was $60 \mathrm{~min}$ to ensure a good signal to noise ratio for the weakly scattering diluted solution.

We define our coordinate system as follows: The $X$ axis is oriented parallel to the incoming direct beam and oriented in the beam direction. The $Z$ axis is chosen vertical and points upwards (normal to the sample surface) and, as a result, the $Y$ axis is lying in the plane of the sample, normal to the direct beam. The incident angle is noted $\alpha_{i}$, and a given exit beam is defined by the angle $\alpha_{f}$ it forms with the $X Y$ plane and the angle $\psi$ between its projection in the $X Y$ and the $X$ direction, see Fig. 4 .

In order to identify the characteristic features introduced by each component of the setup, the diffuse scattering was measured in $\mu$ GISAXS configuration for the bare substrate (glass) and a sheet of TOPAS. The signal measured for a complete empty fluidic cell was then measured and compared to these references. Figure 5 shows the 2D images recorded in each case. In the upper central part of each image, at a position corresponding to $\alpha_{i}=\alpha_{f}$ and $\psi=0$, the shadow of a beamstop is visible. Such a setup is required to prevent saturation or even damage of the $2 \mathrm{D}$ detector due to the high intensity of the specular reflected intensity. At lower exit angles $\alpha_{f}$ one can observe the increased diffuse scatter- 

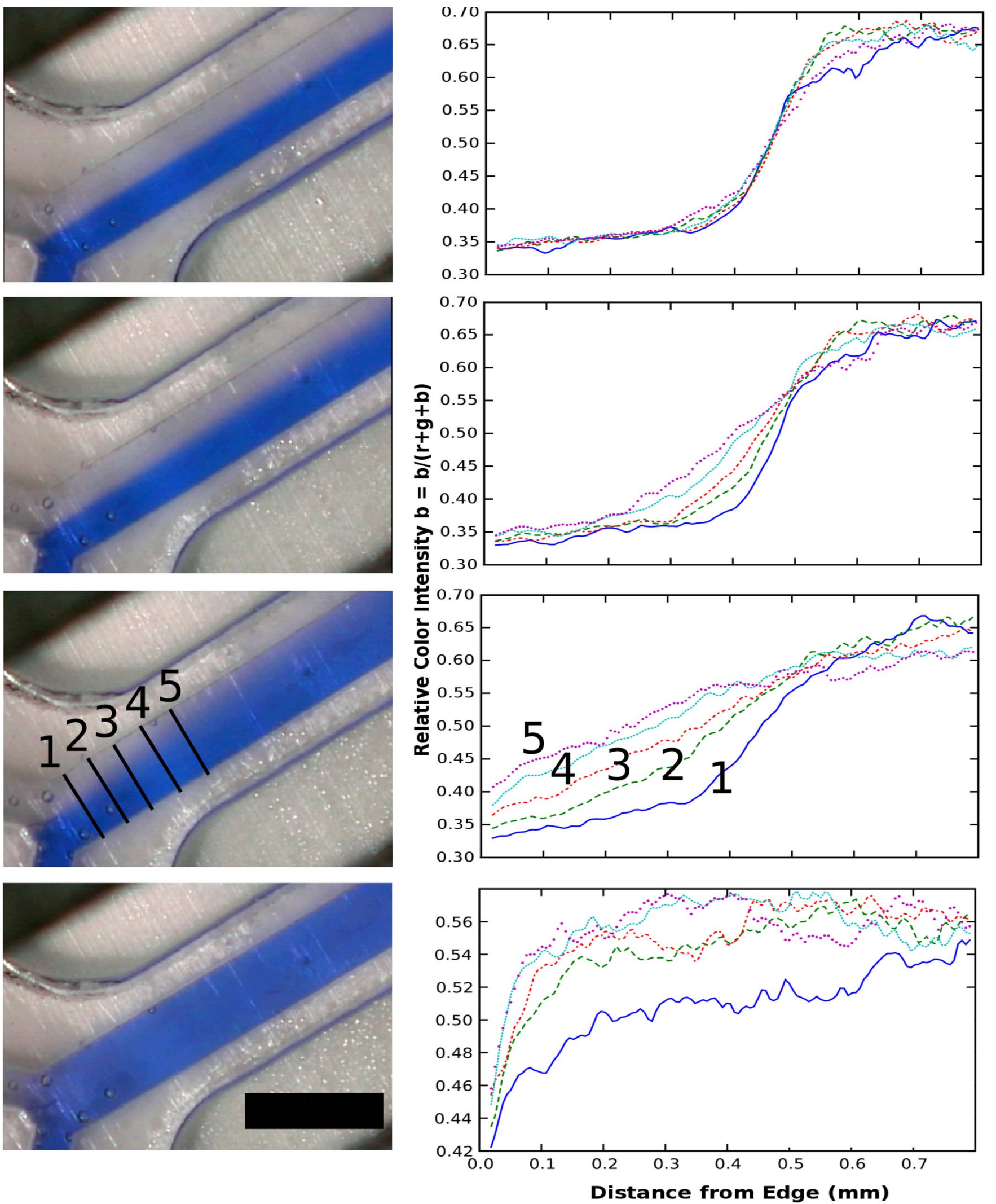

FIG. 3. (Color online) Optical investigation of a mixing experiment. The left column of images shows optical micrograph scale bar is $1 \mathrm{~mm}$. The right column of images shows the profile analysis in terms of relative color intensity blue/(red+green+blue) from the RGB CCD channels at various cutting positions shown as overlay to the optical image (left). From the bottom to the top the flow rate increases $(0.050,0.5,2.0$, and $5.0 \mu \mathrm{m} / \mathrm{s})$.

ing, presenting a maximum intensity at the position of the so-called Yoneda peak. ${ }^{35}$ This peak is located at an exit angle equal to the critical angle of the surface and is thus material dependent.
One can convert the $\mu$ GISAXS pattern into a reciprocal space representation by neglecting the weak dependence in $q_{x}$ and using the following relations: $q_{x}=2 \pi\left(\cos \psi \cos \alpha_{f}\right.$ $\left.-\cos \alpha_{i}\right) / \lambda, \quad q_{y}=2 \pi\left(\sin \psi \cos \alpha_{f}\right) / \lambda, \quad$ and $\quad q_{z}=2 \pi\left(\sin \alpha_{i}\right.$ 


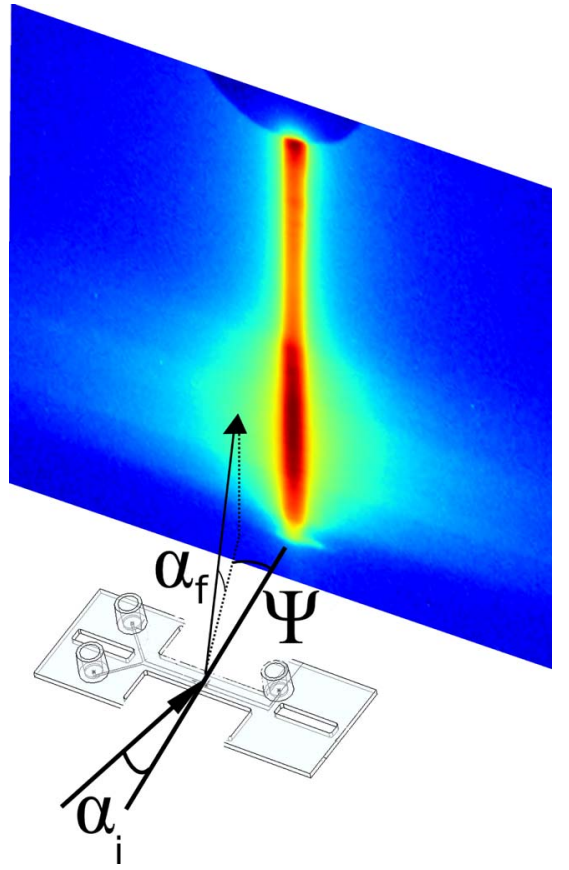

FIG. 4. (Color online) Sketch of the $\mu$ GISAXS geometry.

$\left.+\sin \alpha_{f}\right) / \lambda$. In order to decouple the signals resulting from structural variations parallel to the sample surface and in the direction perpendicular to it, two line cuts through the $2 \mathrm{D}$ $\mu$ GISAXS pattern are typically performed to analyze these images. A vertical cut (so-called detector scan) corresponds to the variation of intensity as a function of $q_{z}$ at a given $q_{y}$. This cut is performed at $q_{y}=0$ and thus yields information about the structure of the sample in the direction perpendicular to the substrate surface. A horizontal cut (so-called out-

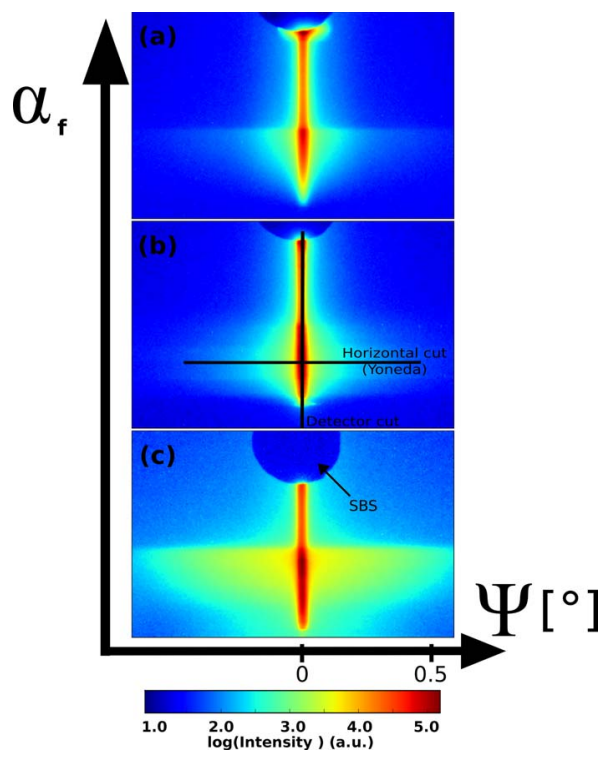

FIG. 5. (Color online) Typical $\mu$ GISAXS patterns (intensity as a function of the exit angle $\alpha_{f}$ and the out-of plane angle $\Psi$ ) recorded with a twodimensional detector for the (a) bare glass substrate, (b) the empty fluidic cell, and (c) the fluidic cell filled with a flowing gold nanoparticle suspension. At the chosen incident angle the Yoneda peak and the specular peak (shadowed by a beamstop, SBS) are well separated along $\alpha_{f}$. The vertical and horizontal lines in Fig. 4(b) indicate the position of the detector and horizontal (out-of-plane) scans.

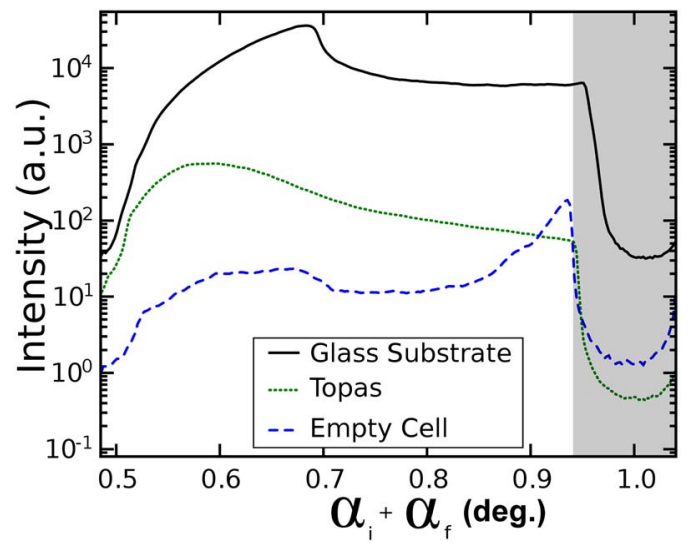

FIG. 6. (Color online) Vertical cuts from the 2D GISAXS pattern shown as a function of the detector angle $\alpha_{i}+\alpha_{f}$ for the (a) bare glass substrate, (b) a flat sheet of TOPAS, and (c) the empty fluidic cell.

of-plane scan) yields information about the intensity variations as a function of $q_{y}$ for a given $q_{z}$ and thus reveals correlations in the direction parallel to the sample surface. This cut is usually performed at $q_{z}$ values corresponding to the maximum sensitivity for a given component of the sample. It corresponds to an exit angle equal to the critical angles of the material under consideration, i.e., to the corresponding Yoneda peak position. ${ }^{30}$

Figure 6 shows the vertical cuts at $q_{y}=0$ obtained for the bare glass substrate, a foil of TOPAS, and assembled empty cell (containing glass and TOPAS). The dark gray region around $\alpha_{i}+\alpha_{f}=1^{\circ}$ indicates the region which was shadowed in the $\mu$ GISAXS measurements by the specular beamstop. At smaller exit angles the Yoneda peaks of the different materials are observed. The bare glass substrate shows a well defined, relatively sharp peak, as expected for bare glass. The TOPAS sample shows a broader peak located at smaller exit angles. This observation is expected because the critical angle of glass is larger than that of polyolefins due to the larger electron density of glass. The vertical cut obtained for a complete empty cell is roughly a superposition of the ones obtained for its constituting elements and no additional strong feature is observed in the diffuse scattering region. As a consequence, the designed fluidic cell is well suited for GISAXS experiments and will not dominate the observed diffuse scattering.

\section{Injection of gold nanoparticles}

Once established that the cell itself introduces very little additional contribution to the diffuse scattering, we injected a water based gold nanoparticle suspension (nominal mean particle size of $20 \mathrm{~nm}$ and concentration of 7 $\times 10^{11}$ particles $/ \mathrm{ml}$ from $\mathrm{G}$. Kisker $\mathrm{GmbH}$, Germany) in the channel in order to test if this setup enables to detect lateral structures at the substrate/flowing liquid interface. During this experiment, the flow rate was kept at a value of $166 \mu \mathrm{m} / \mathrm{s}$. Gold nanoparticles are very well suited to test the performance of our GISAXS setup because they provide a water based monodisperse system of strong scatterers in the nanometer range. Previous measurements performed on sput- 


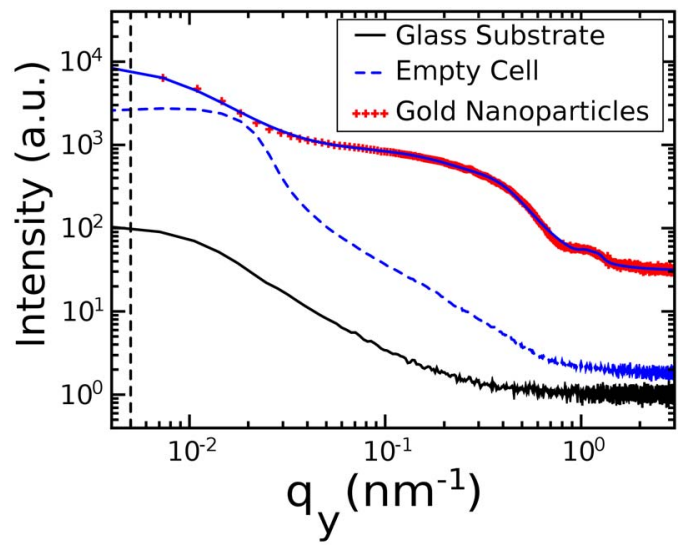

FIG. 7. (Color online) Double logarithmic plot of the horizontal line cuts from the 2D GISAXS pattern for the (a) bare glass substrate, (b) the empty fluidic cell, and (c) the fluidic cell filled with a flowing gold nanoparticle suspension. A model fitting of the gold scattering is shown as overlay (continuous line). The vertical dashed line locates the resolution limit of the measurement.

tered gold thin films have shown that it is possible to resolve the scattering form factor of such particles and to characterize their structural correlations. ${ }^{36}$

Figure 5(c) is the image showing the 2D $\mu$ GISAXS pattern recorded during this experiment. One clearly sees the very strong wings developing in the $q_{y}$ direction in the scattering pattern. These are the sign of density fluctuations in the horizontal plane caused by the gold nanoparticles. These fluctuations can be characterized via a horizontal cut performed at the maximum of the Yoneda peak. For comparison, Fig. 7 shows this cut as well as those obtained for the bare glass substrate and the empty cell. The curves measured for the substrate and empty cell show only a very little scattering, which decays simply with increasing $q_{y}$. In contrast, the scattering of the gold nanoparticle solution displays a strong signal around $0.25 \mathrm{~nm}^{-1}$ and a weaker peak around $1 \mathrm{~nm}^{-1}$.

A simple model fitting of this profile was performed using stretched Lorentzian peaks, and is shown as an overlay to the experimental data in Fig. 7. The central line corresponds to the so-called resolution peak and the higher $q$ peaks are characteristic of the surface structure. Peaks are observed at $q=0.02,0.240$, and $1 \mathrm{~nm}^{-1}$, corresponding in direct space to characteristic lengths of 314,26 , and $6 \mathrm{~nm}$, respectively. The first broad peak is attributed to the diffuse scattering observed for the empty cell. Since the nominal particle diameter used in this experiment is $20 \mathrm{~nm}$, the signals at $26 \mathrm{~nm}$ can clearly be attributed to a nearest neighbor distance for the nanoparticles at the interface. The second peak is attributed to the form factor of the nanoparticles.

These observations clearly demonstrate that using our setup, we obtained a signal characteristic of the solid-liquid interface in a flowing system.

\section{CONCLUSIONS AND PERSPECTIVES}

We have demonstrated a fluidic setup optimized for microbeam GISAXS characterization of lateral structures at the liquid/substrate interface. This setup allows to simultaneously record the diffuse scattering pattern stemming from the interface and to monitor the flow by optical microscopy. With the designed setup, mixing studies can be performed over a broad range of flow rates. In a first demonstrative test experiment with this fluidic cell we were able to detect the presence of gold nanoparticles in flowing aqueous solution at the interface of a glass substrate. To our knowledge, this is the first time that such a result is achieved. This setup will open new perspectives for the study of microfluidics and buried liquid-solid interfaces. The extensions we envision for this work are the use of various substrates and the investigation of the surface treatment effect upon flow and aggregation at the interface. Downscaling of this device is possible to a certain extent and would be useful when x-ray beams, focused down to a smaller diameter, would be used in order to get a spatial resolution in the range of micron and even some tens of nanometers.

\section{ACKNOWLEDGMENTS}

We thank A. Timmann for the support during the setup of the beamline BW4 and E. Metwalli and W. Wang for their help during the scattering experiments. This work was financially supported by DFG in the priority program for nanoand microfluidics SPP1164 (Grant No. MU1487/2).

${ }^{1}$ E. Delamarche, H. Bernard, H. Schmid, B. Michel, and H. Biebuyck, Science 276, 779 (1997).

${ }^{2}$ B. H. Weigl and P. Yager, Science 283, 346 (1999).

${ }^{3}$ P. S. Dittrich and P. Schwille, Anal. Chem. 75, 5767 (2003).

${ }^{4}$ T. Thorsen, S. J. Maerkl, and S. R. Quake, Science 298, 5593 (2002).

${ }^{5}$ R. F. Ismagilov, J. M. K. Ng, P. J. A. Kenis, and G. M. Whitesides, Anal. Chem. 73, 5207 (2001).

${ }^{6}$ M. A. Unger, H.-P. Chou, T. Thorsen, A. Scherer, and S. R. Quake, Science 288, 113 (2000).

${ }^{7}$ N. Nguyen, X. Huang, and T. K. Chuan, J. Fluids Eng. 124, 384 (2002).

${ }^{8}$ H. Cao, J. O. Tegenfeldt, R. H. Austin, and S. Y. Chou, Appl. Phys. Lett. 81, 3058 (2002).

${ }^{9}$ P. Müller-Buschbaum, E. Bauer, E. Maurer, K. Schlögl, S. V. Roth, and R. Gehrke, Appl. Phys. Lett. 88, 083114 (2006).

${ }^{10}$ E. Thrusha, O. Levia, L. J. Cooka, J. Deichb, A. Kurtzb, S. J. Smithc, W. E. Moernerb, and J. S. Harris, Jr., Sens. Actuators B 105, 393 (2005).

${ }^{11}$ J. D. Tice, H. Song, A. D. Lyon, and R. F. Ismagilov, Langmuir 19, 9127 (2003).

${ }^{12}$ D. Belder, Angew. Chem. 7, 3587 (2005).

${ }^{13}$ A. D. Strook, S. K. W. Dertinger, A. Ajdari, I. Mesic, H. A. Stone, and G. M. Whitesides, Science 295, 647 (2002).

${ }^{14}$ B. S. Broyles, S. C. Jacobsen, and J. M. Ramsey, Anal. Chem. 75, 2761 (2003).

${ }^{15}$ R. P. Castro, Y. Cohen, and H. G. Monbouquette, J. Membr. Sci. 1, 82 (1996).

${ }^{16} \mathrm{H}$. Zhang and Y. Ito, Handbook of Polyelectrolytes and Their Applications (American Scientific, Los Angels, CA, 2002), Vol. 1, Chap. 8, pp. 183206.

${ }^{17}$ P. S. Doyle, J. Bibette, A. Bancaud, and J.-L. Viovy, Science 295, 2237 (2002).

${ }^{18}$ T. M. Squires and R. Quake, Rev. Mod. Phys. 77, 977 (2005).

${ }^{19}$ D. R. Reyes, D. Iossifidis, P. A. Auroux, and A. Manz, Anal. Chem. 74, 2623 (2002).

${ }^{20}$ G. Glatter and O. Kratky, Small Angle X-Ray Scattering (Academic, New York, 1982).

${ }^{21}$ Ma. Kisilak, H. Anderson, N. S. Babcock, M. R. Stetzer, and S. H. J. Idziaka, Rev. Sci. Instrum. 72, 4305 (2001).

${ }^{22}$ J. Lipfert, I. S. Millett, S. Seifert, and S. Doniach, Rev. Sci. Instrum. 77, 046108 (2006)

${ }^{23}$ C. E. Miller, J. Majewski, T. Gog, and T. L. Kuhl, Phys. Rev. Lett. 94, 238104 (2005)

${ }^{24}$ C. J. Yu, A. G. Richter, A. Datta, M. K. Durbin, and P. Dutta, Phys. Rev. Lett. 82, 2326 (1999).

${ }^{25}$ J. R. Levine, J. B. Cohen, Y. W. Chung, and P. Georgopoulos, J. Appl. 
Crystallogr. 22, 528 (1989).

${ }^{26}$ T. Salditt, T. H. Metzger, J. Peisl, and G. Goerigk, J. Phys. D 28, A236 (1995).

${ }^{27}$ P. Müller-Buschbaum, P. Vanhoorne, V. Scheumann, and M. Stamm, Europhys. Lett. 40, 655 (1997)

${ }^{28}$ A. Naudon, D. Babonneau, D. Thiaudiere, and S. Lequien, Physica B 283, 69 (2000).

${ }^{29}$ J. A. Nielsen and D. Mc. Morrow, Elements of Modern X-Ray Physics (Wiley, New York, 2001).

${ }^{30}$ P. Müller-Buschbaum, Anal. Bioanal. Chem. 376, 3 (2003).

${ }^{31}$ S. V. Roth, R. Döhrmann, M. Dommach, M. Kuhlmann, I. Kröger, R.
Gehrke, H. Walter, C. Schroer, B. Lengeler, and P. Müller-Buschbaum, Rev. Sci. Instrum. 77, 085106 (2006).

${ }^{32}$ A. Snigirev, V. Kohn, I. Snigireva, A. Souvorov, and B. Lengeler, Appl. Opt. 37, 653 (1998).

${ }^{33}$ C. Reich, M. Hochrein, B. Krause, and B. Nickel, Rev. Sci. Instrum. 76, 095103 (2005).

${ }^{34}$ P. Müller-Buschbaum, Eur. Phys. J. E 12, 443 (2003).

${ }^{35}$ Y. Yoneda, Phys. Rev. 131, 2010 (1963).

${ }^{36}$ S. V. Roth, H. Walter, M. Burghammer, C. Riekel, B. Lengeler, C. Schroer, M. Kuhlmann, T. Walther, A. Sehrbrock, and P. MüllerBuschbaum, Appl. Phys. Lett. 88, 021910 (2006). 